\title{
An Unusual Reverse Turn Structure Adopted by a Furanoid Sugar Amino Acid Incorporated in Gramicidin S
}

\author{
Gijsbert M. Grotenbreg, Mattie S. M. Timmer, Antonio L. Llamas-Saiz, Martijn Verdoes, Gijsbert A. van der \\ Marel, Mark J. van Raaij, Herman S. Overkleeft and Mark Overhand
}

\section{Supporting Information :}

Contents :

$\begin{array}{lll}\text { A } & \text { General } & \text { S1 } \\ \text { B } & \text { Experimental Procedures of the Sugar Amino Acid Synthesis } & \text { S2 - S3 } \\ \text { C } & \text { Experimental Procedures of the Peptide Synthesis } & \text { S4 - S6 } \\ \text { D } & \text { X-ray crystallographic data } & \text { S7 - S8 }\end{array}$

\section{A. General :}

All reactions were performed under an inert atmosphere and at ambient temperature unless stated otherwise. Reactions were monitored by TLC-analysis using DC-fertigfolien (Schleicher \& Schuell, F1500, LS254) with detection by spraying with $20 \% \mathrm{H}_{2} \mathrm{SO}_{4}$ in ethanol followed by charring at $\sim 150^{\circ} \mathrm{C}$ or by spraying with a solution of $\left(\mathrm{NH}_{4}\right)_{6} \mathrm{Mo}_{7} \mathrm{O}_{24} \cdot 4 \mathrm{H}_{2} \mathrm{O}(25 \mathrm{~g} / \mathrm{L})$ and $\left(\mathrm{NH}_{4}\right)_{4} \mathrm{Ce}\left(\mathrm{SO}_{4}\right)_{4} \cdot 2 \mathrm{H}_{2} \mathrm{O}(10 \mathrm{~g} / \mathrm{L})$ in $10 \%$ sulfuric acid followed by charring at $\sim 150^{\circ} \mathrm{C}$. Column chromatography was performed on Merck silicagel $(0.040-0.063 \mathrm{~nm})$ and size exclusion chromatography on Sephadex ${ }^{\mathrm{TM}} \mathrm{LH}-20$.

Mass spectra were recorded on a PE/Sciex API 165 instrument with a custom-build Electrospray Ionisation (ESI) interface and HRMS (SIM mode) were recorded on a TSQ Quantum (Thermo Finnigan) fitted with an accurate mass option, interpolating between PEG-calibration peaks. For LC/MS analysis, a Jasco HPLCsystem (detection simultaneously at 214 and $254 \mathrm{~nm}$ ) equipped with an analytical Alltima $\mathrm{C}_{18}$ colomn (Alltech, $4.6 \mathrm{mmD} \times 250 \mathrm{mmL}, 5 \mu$ particle size) in combination with buffers $\mathrm{A}: \mathrm{H}_{2} \mathrm{O}, \mathrm{B}: \mathrm{MeCN}$ and $\mathrm{C}: 0.5 \%$ aq. TFA and coupled to a Perkin Elmer Sciex API 165 mass instrument with a custom-made Electronspray Interface (ESI) was used. For RP-HPLC purification of the peptide, a BioCAD "Vision" automated HPLC system (PerSeptiveBiosystems, inc.) supplied with a semi-preperative Alltima $\mathrm{C}_{18}$ column (Alltech, 10.0 $\mathrm{mmD} \times 250 \mathrm{mmL}, 5 \mu$ particle size) was used. The applied buffers were $\mathrm{A}: \mathrm{H}_{2} \mathrm{O}, \mathrm{B}: \mathrm{MeCN}$ and $\mathrm{C}: 1.0 \%$ aq. TFA.

${ }^{1} \mathrm{H}$ - and ${ }^{13} \mathrm{C}$-APT-NMR spectra were recorded on a Brüker AV-400 (400/100 MHz) and the peptide 7 was analyzed using a Brüker DMX 600 spectrometer equipped with a pulsed field gradient accessory. Chemical shifts are given in ppm $(\delta)$ relative to tetramethylsilane as internal standard $\left({ }^{1} \mathrm{H}-\mathrm{NMR}\right)$ or $\mathrm{CDCl}_{3}\left({ }^{13} \mathrm{C}-\mathrm{NMR}\right)$. Coupling constants are given in $\mathrm{Hz}$. All presented ${ }^{13} \mathrm{C}$-APT spectra are proton decoupled. Optical rotations were measured on a Propol automatic polarimeter (Sodium D line, $\lambda=589 \mathrm{~nm}$ ) and ATR-IR spectra were recorded on a Shimadzu FTIR-8300 fitted with a single bounce DurasamplIR diamond crystal ATR-element. 


\section{B. Experimental Procedures of the Sugar Amino Acid Synthesis}

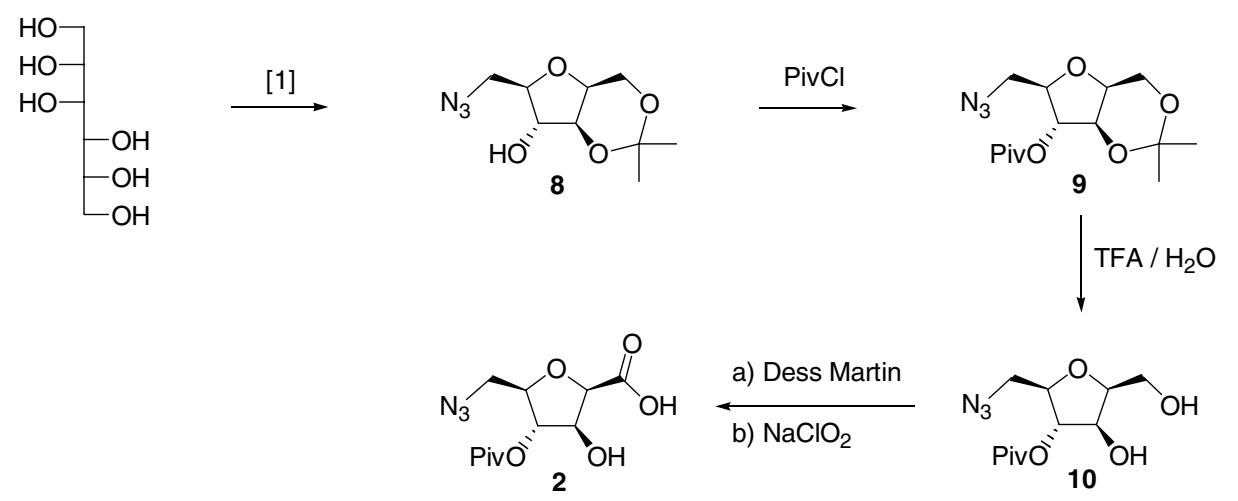

Scheme 1: Synthesis of the Sugar Amino Acid

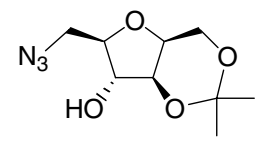

2,5-Anhydro-6-azido-6-deoxy-1,3-O-isopropylidene-D-glucitol (8)

Prepared as described by Timmer et al. ${ }^{1}$

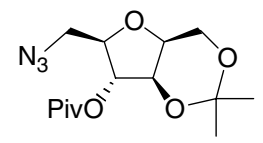

2,5-Anhydro-6-azido-6-deoxy-1,3- $O$-isopropylidene-4- $O$-pivaloyl-D-glucitol (9)

Azide 8 (100 mmol, $22.9 \mathrm{~g})$ was coevaporated twice with pyridine and dissolved in pyridine $(500 \mathrm{~mL})$. After pivaloylchloride $(\mathrm{PivCl})(1.2$ equiv., $120 \mathrm{mmol}, 14.7 \mathrm{~mL})$ was added, the reaction mixture was stirred overnight before being concentrated. The residue was dissolved in EtOAc and washed with $1 \mathrm{M}$ aq. $\mathrm{HCl}$, water and brine. The EtOAc layer was dried over $\mathrm{MgSO}_{4}$ and concentrated. Silica colomn chromatography (20\% EtOAc in light PE) yielded fully protected glucitol 9 quantitatively $(100 \mathrm{mmol}, 31.4 \mathrm{~g})$ as a transparant oil. ${ }^{1} \mathrm{H}-\mathrm{NMR}\left(400 \mathrm{MHz}, \mathrm{CDCl}_{3}\right): \delta 4.85(\mathrm{~d}, 1 \mathrm{H}$, $\left.\mathrm{H}_{4}, J_{4,5}=1.7 \mathrm{~Hz}\right), 4.25\left(\mathrm{~d}, 1 \mathrm{H}, \mathrm{H}_{3}, J_{3,2}=2.6 \mathrm{~Hz}\right), 4.10\left(\mathrm{dd}, 1 \mathrm{H}, \mathrm{H}_{1 \mathrm{a}}, J_{1 \mathrm{a}, 2}=2.5 \mathrm{~Hz}, J_{1 \mathrm{a}, 1 \mathrm{~b}}=13.4 \mathrm{~Hz}\right), 4.03(\mathrm{dd}$, $\left.1 \mathrm{H}, \mathrm{H}_{1 \mathrm{~b}}, J_{1 \mathrm{~b}, 2}=1.6 \mathrm{~Hz}, J_{1 \mathrm{~b}, 1 \mathrm{a}}=13.4 \mathrm{~Hz}\right), 3.98\left(\mathrm{ddd}, 1 \mathrm{H}, \mathrm{H}_{5}, J_{5,4}=1.7 \mathrm{~Hz}, J_{5,6 \mathrm{~b}}=4.7 \mathrm{~Hz}, J_{5,6 \mathrm{a}}=7.8 \mathrm{~Hz}\right), 3.89$ $\left(\mathrm{ddd}, 1 \mathrm{H}, \mathrm{H}_{2}, J_{2,1 \mathrm{~b}}=1.6 \mathrm{~Hz}, J_{2,1 \mathrm{a}}=J_{2,3}=2.6 \mathrm{~Hz}\right), 3.66\left(\mathrm{dd}, 1 \mathrm{H}, \mathrm{H}_{6 \mathrm{a}}, J_{6 \mathrm{a}, 5}=7.8 \mathrm{~Hz}, J_{6 \mathrm{a}, 6 \mathrm{~b}}=12.6 \mathrm{~Hz}\right), 3.50$ (dd, $\left.1 \mathrm{H}, \mathrm{H}_{6 \mathrm{~b}}, J_{6 \mathrm{~b}, 5}=4.7 \mathrm{~Hz}, J_{6 \mathrm{~b}, 6 \mathrm{a}}=12.6 \mathrm{~Hz}\right), 1.44\left(\mathrm{~s}, 3 \mathrm{H}, \mathrm{CH}_{3} i \mathrm{Pr}\right), 1.41\left(\mathrm{~s}, 3 \mathrm{H}, \mathrm{CH}_{3} i \mathrm{Pr}\right), 1.20\left(\mathrm{~s}, 9 \mathrm{H}, 3 \times \mathrm{CH}_{3} \mathrm{Piv}\right)$ ${ }^{13} \mathrm{C}-\mathrm{NMR}\left(100 \mathrm{MHz}, \mathrm{CDCl}_{3}\right): \delta 177.2(\mathrm{C}=\mathrm{O} \mathrm{Piv}), 97.6\left(\mathrm{C}_{\mathrm{q}} i \mathrm{Pr}\right), 83.6\left(\mathrm{C}_{5}\right), 80.1\left(\mathrm{C}_{4}\right), 73.6\left(\mathrm{C}_{3}\right), 73.3\left(\mathrm{C}_{2}\right)$, $60.1\left(\mathrm{C}_{1}\right), 52.7\left(\mathrm{C}_{6}\right), 38.5\left(\mathrm{C}_{\mathrm{q}} \mathrm{Piv}\right), 28.7\left(\mathrm{CH}_{3} \mathrm{iPr}\right), 26.9\left(\mathrm{CH}_{3} \mathrm{Piv}\right), 18.8\left(\mathrm{CH}_{3}\right.$ iPr). ATR-IR (thin film) 2977.9, 2096.5, 1732.0, 1481.2, 1375.2, 1280.6, 1143.7, 1091.6, 929.6, 846.7 $\mathrm{cm}^{-1} .[\alpha]_{\mathrm{D}}^{23}+22.4\left(\mathrm{c}=1.00, \mathrm{CHCl}_{3}\right)$ MS (ESI): $m / z, 314.3[\mathrm{M}+\mathrm{H}]^{+}, 336.1[\mathrm{M}+\mathrm{Na}]^{+}$. HRMS: calcd for $\mathrm{C}_{14} \mathrm{H}_{23} \mathrm{~N}_{3} \mathrm{O}_{5} \mathrm{NH}_{4}$ 331.1981, found 331.1968.

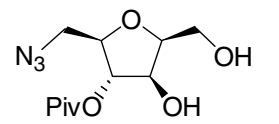

\section{2,5-Anhydro-6-azido-6-deoxy-4-O-pivaloyl-D-glucitol (10)}

Glucitol 9 (100 mmol, $31.4 \mathrm{~g})$ was dissolved in a mixture of water/TFA (400 mL, 2/1, v/v). The resulting white suspension was stirred overnight to give a homogeneous yellow solution. The reaction mixture was concentrated and coevaporated with toluene before being purified by column chromatography (toluene $\rightarrow 30 \%$ EtOAc in toluene) furnishing the title compound 10 (17.2 g, 63 mmol, $63 \%)$ as a transparant oil. ${ }^{1} \mathrm{H}-\mathrm{NMR}\left(400 \mathrm{MHz}, \mathrm{CDCl}_{3}\right): \delta 4.81\left(\mathrm{dd}, 1 \mathrm{H}, \mathrm{H}_{4}, J_{4,3}=1.8 \mathrm{~Hz}, J_{4,5}=3.4 \mathrm{~Hz}\right)$, $4.28\left(\mathrm{dd}, 1 \mathrm{H}, \mathrm{H}_{3}, J_{3,4}=1.8 \mathrm{~Hz}, J_{3,2}=4.3 \mathrm{~Hz}\right), 4.05\left(\mathrm{dd}, 1 \mathrm{H}, \mathrm{H}_{2}, J_{2,3}=2.5 \mathrm{~Hz}, J_{2,1}=8.6 \mathrm{~Hz}\right), 3.97\left(\mathrm{~m}, 3 \mathrm{H}, \mathrm{H}_{5}\right.$ and $\left.2 \times \mathrm{H}_{1}\right) 3.63\left(\mathrm{~d}, 2 \mathrm{H}, 2 \times \mathrm{H}_{6}, J_{6,5}=4.8 \mathrm{~Hz}\right), 1.20\left(\mathrm{~s}, 9 \mathrm{H}, 3 \times \mathrm{CH}_{3}\right.$ Piv) ${ }^{13} \mathrm{C}-\mathrm{NMR}\left(100 \mathrm{MHz}, \mathrm{CDCl}_{3}\right): \delta$ 178.3 (C=O Piv), $81.8\left(\mathrm{C}_{5}\right), 81.5\left(\mathrm{C}_{4}\right), 80.9\left(\mathrm{C}_{2}\right), 76.5\left(\mathrm{C}_{3}\right), 61.0\left(\mathrm{C}_{1}\right), 52.4\left(\mathrm{C}_{6}\right), 38.5\left(\mathrm{C}_{\mathrm{q}} \mathrm{Piv}\right), 26.8\left(\mathrm{CH}_{3} \mathrm{Piv}\right)$. ATR-IR (thin film) 3328.9, 2974.0, 2098.4, 1726.2, 1481.2, 1280.6, 1149.5, 1078.1, $1035.7 \mathrm{~cm}^{-1} \cdot[\alpha]_{\mathrm{D}}^{23}+41.6$ $\left(\mathrm{c}=1.00, \mathrm{CHCl}_{3}\right) \mathrm{MS}(\mathrm{ESI}): \mathrm{m} / z 273.9[\mathrm{M}+\mathrm{H}]^{+}, 296.2[\mathrm{M}+\mathrm{Na}]^{+}$. HRMS: calcd for $\mathrm{C}_{11} \mathrm{H}_{19} \mathrm{~N}_{3} \mathrm{O}_{5} \mathrm{H}$ 274.1403, found 274.1409. 


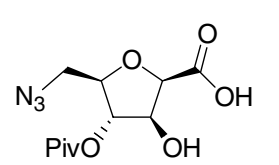

\section{2,5-Anhydro-6-azido-6-deoxy-4-O-pivaloyl-D-gluconic acid (2).}

Diol 10 (5.8 g, $20 \mathrm{mmol}$ ) was coevaporated twice with toluene, dissolved in DCM (100 $\mathrm{mL}$ ), placed under an argon atmosphere and cooled to $0^{\circ} \mathrm{C}$, before Dess-Martin periodinane (1.1 equiv., $9.35 \mathrm{~g}, 22 \mathrm{mmol}$ ) was added under vigorous stirring. The reaction mixture was stirred for $30 \mathrm{~min}$. before a sat. aq. $\mathrm{NaS}_{2} \mathrm{O}_{3} /$ sat. aq. $\mathrm{NaHCO}_{3}$ solution $\left.(100 \mathrm{~mL}, 7 / 3 \mathrm{(v/v})\right)$ was added and stirred for an additional $15 \mathrm{~min}$. Then, the DCM layer was separated, washed with $\mathrm{H}_{2} \mathrm{O}$ and brine, dried over $\mathrm{MgSO}_{4}$ and concentrated. The residue was coevaporated with toluene and purified by column chromatography (toluene $\rightarrow 5 \%$ (v/v) EtOAc in toluene) yielding 6-azido-6-deoxy-4-pivaloyl-2,5-anhydroD-glucose $(4.97 \mathrm{~g}, 18.3 \mathrm{mmol}, 91.5 \%)$. The aldehyde $(2.56 \mathrm{~g}, 9.4 \mathrm{mmol})$ was dissolved in a solution of tertbutanol $(80 \mathrm{~mL}), 2$-methyl-2-butene $(20 \mathrm{~mL})$ and water $(80 \mathrm{~m})$, before $\mathrm{NaH}_{2} \mathrm{PO}_{4}(8.0 \mathrm{~g})$ and $\mathrm{NaClO}_{2}(8.0 \mathrm{~g})$ were added. The reaction was stirred overnight, before the solution was acidified and extracted with EtOAc (2 $\times$ ). The EtOAc layers were dried over $\mathrm{MgSO}_{4}$, concentrated and purified by column chromatography (toluene $\rightarrow 1 \% \mathrm{AcOH}$ in EtOAc) yielding the title compound 2 (1.55 g, $5.38 \mathrm{mmol}, 57 \%$ (52 \%, 2 steps)). ${ }^{1} \mathrm{H}-\mathrm{NMR}$ $\left(400 \mathrm{MHz}, \mathrm{CDCl}_{3}\right): \delta 4.86\left(\mathrm{dd}, 1 \mathrm{H}, \mathrm{H}_{4}, J_{4,3}=1.0 \mathrm{~Hz}, J_{4,5}=2.0 \mathrm{~Hz}\right), 4.58\left(\mathrm{~d}, 1 \mathrm{H}, \mathrm{H}_{2}, J_{2,3}=4.0 \mathrm{~Hz}\right), 4.41(\mathrm{dd}$, $\left.1 \mathrm{H}, \mathrm{H}_{3}, J_{3,4}=1.0 \mathrm{~Hz}, J_{3,2}=4.0 \mathrm{~Hz}\right), 4.00\left(\mathrm{ddd}, 1 \mathrm{H}, \mathrm{H}_{5}, J_{5,4}=2.0 \mathrm{~Hz}, J_{5,6 \mathrm{~b}}=4.3 \mathrm{~Hz}, J_{5,6 \mathrm{a}}=5.5 \mathrm{~Hz}\right), 3.73(\mathrm{dd}, 1 \mathrm{H}$, $\left.\mathrm{H}_{6 \mathrm{a}}, J_{6 \mathrm{a}, 5}=5.5 \mathrm{~Hz}, J_{6 \mathrm{a}, 6 \mathrm{~b}}=12.8 \mathrm{~Hz}\right), 3.63\left(\mathrm{dd}, 1 \mathrm{H}, \mathrm{H}_{6 \mathrm{~b}}, J_{6 \mathrm{~b}, 5}=4.3 \mathrm{~Hz}, J_{6 \mathrm{~b}, 6 \mathrm{a}}=12.8 \mathrm{~Hz}\right), 1.15\left(\mathrm{~s}, 9 \mathrm{H}, 3 \times \mathrm{CH}_{3}\right.$ Piv). ${ }^{13} \mathrm{C}-\mathrm{NMR}\left(100 \mathrm{MHz}, \mathrm{CDCl}_{3}\right): \delta 177.9$ (C=O Piv), $171.5(\mathrm{COOH}) 83.3\left(\mathrm{C}_{5}\right), 81.4\left(\mathrm{C}_{2}\right), 80.1\left(\mathrm{C}_{4}\right), 76.0$ $\left(\mathrm{C}_{3}\right), 52.0\left(\mathrm{C}_{6}\right), 38.6\left(\mathrm{C}_{\mathrm{q}} \mathrm{Piv}\right), 26.8\left(\mathrm{CH}_{3}\right.$ Piv). ATR-IR (thin film) 3421.6, 2976.0, 2102.3, 1728.1, 1481.2, 1282.6, 1143.7, 1097.4, $1037.6 \mathrm{~cm}^{-1} .[\alpha]_{\mathrm{D}}^{23}+30.0\left(\mathrm{c} 1.00, \mathrm{CHCl}_{3}\right)$. MS (ESI): $\mathrm{m} / z 288.2[\mathrm{M}+\mathrm{H}]^{+}, 310.1$ $[\mathrm{M}+\mathrm{Na}]^{+}$. HRMS: calcd for $\mathrm{C}_{11} \mathrm{H}_{17} \mathrm{~N}_{3} \mathrm{O}_{6} \mathrm{H}$ 288.1196, found 288.1240. 


\section{Experimental Procedures of the Peptide Synthesis}

\section{Fmoc-Leu-HMPB-O Fmoc-Leu-HMPB-MBHA resin (3)}

Commercially available 4-methylbenzhydrylamine (MBHA) functionalized polystyrene resin $(2.22 \mathrm{~g}, 0.9 \mathrm{mmol} / \mathrm{g}, 2.0 \mathrm{mmol})$ was shaken with NMP (30 mL, $3 \times, 3 \mathrm{~min}$.) followed by addition of a pre-activated mixture of 4-(4-hydroxymethyl-3-methoxyphenoxy)-butyric acid (HMPB) (3 equiv., 1.44 g, 6.0 mmol), benzotriazole-1-yloxy-tris(dimethylamino)phosphonium hexafluorophosphate (BOP) (3 equiv., 2.652 $\mathrm{g}, 6.0 \mathrm{mmol}$ ) and $N, N$-diisopropylethylamine (DiPEA) (6 equiv., $2.09 \mathrm{~mL}, 12.0 \mathrm{mmol})$ in NMP $(25 \mathrm{~mL})$. Shaking was continued overnight after wich the resin was washed with NMP (30 mL, $3 \times, 3 \mathrm{~min}$.) and DCM (30 $\mathrm{mL}, 3 \times, 3 \mathrm{~min}$.). Next, the resin was transferred to a flask, coevaporated with DCE $(30 \mathrm{~mL}, 3 \times)$ and condensed with Fmoc-Leu-OH (3 equiv., $2.12 \mathrm{~g}, 6.0 \mathrm{mmol})$ under de influence of $N, N^{\prime}-$ diisopropylcarbodiimide (DIC) $(3.3$ equiv., $1.03 \mathrm{~mL}, 6.6 \mathrm{mmol})$ and a catalytic amount of 4dimethylaminopyridine (DMAP) $(40 \mathrm{mg}, 0.33 \mathrm{mmol})$ for two hours. The resin was then filtered and washed with $\mathrm{DCM}(30 \mathrm{~mL}, 3 \times, 3 \mathrm{~min}$.) and subjected to a second condensation sequence, gaining fully loaded resin 3. The loading of the resin was determined to be $0.50 \mathrm{mmol} / \mathrm{g}$ by spectrophotometric analysis.

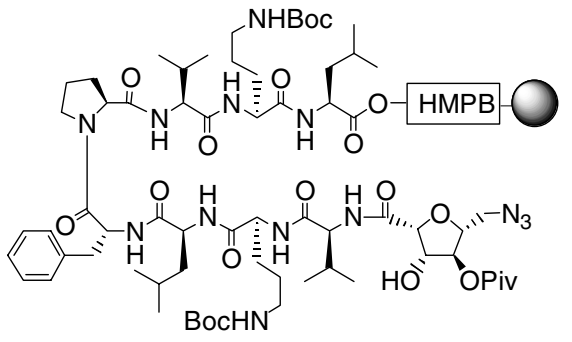

SAA(Piv)-Val-Orn(Boc)-Leu- ${ }^{D}$ Phe-Pro-Val-Orn(Boc)-Leu-HMPBMBHA resin (4)

Resin $3(0.2 \mathrm{~g}, 0.5 \mathrm{mmol} / \mathrm{g}, 0.1 \mathrm{mmol})$ was submitted to seven cycles of Fmoc solid-phase synthesis with Fmoc-Orn 8 (Boc)-OH, Fmoc-Val $\mathrm{OH}, \quad \mathrm{Fmoc}_{-} \mathrm{PrO}_{6}-\mathrm{OH}, \quad$ Fmoc- ${ }^{\mathrm{D}} \mathrm{Phe}_{5}-\mathrm{OH}, \quad \mathrm{Fmoc}_{-} \mathrm{Leu}_{4}-\mathrm{OH}$, Fmoc$\mathrm{Orn}_{3}(\mathrm{Boc})-\mathrm{OH}$ and $\mathrm{Fmoc}-\mathrm{Val}_{2}-\mathrm{OH}$, respectively, as follows: a) deprotection with piperidine / NMP (1/4, v/v, $5 \mathrm{~mL}, 15 \mathrm{~min}$.); b) wash with NMP (5 mL, $3 \times, 3 \mathrm{~min}$.); c) coupling of the appropriate Fmoc

amino acid (2.5 equiv., $0.25 \mathrm{mmol})$ in the presence of BOP (2.5 equiv., $0.25 \mathrm{mmol}, 0.11 \mathrm{~g}), \mathrm{N}$ hydroxybenzotriazole (HOBt, 2.5 equiv., $0.25 \mathrm{mmol}, 34 \mathrm{mg}$ ) and DiPEA (3 equiv., $0.3 \mathrm{mmol}, 0.051 \mathrm{~mL}$ ) which was preactivated for $2 \mathrm{~min}$. in NMP ( $5 \mathrm{~mL}$ ) and shaken for $90 \mathrm{~min}$.; d) wash with NMP (5 mL, 3×, 3 min.). Couplings were monitored for completion by the Kaiser test. ${ }^{2}$ Finally, the $N$-terminal amine was liberated by Fmoc-deprotection with piperidine / NMP (1/4, v/v, $5 \mathrm{~mL}, 15 \mathrm{~min}$.) followed by washing with NMP ( $5 \mathrm{~mL}, 3 \times, 3 \mathrm{~min}$.). To the resin bound octapeptide, a preactivated solution of SAA 2 (3.6 equiv., 105 $\mathrm{mg}, 0.366 \mathrm{mmol}$ ), BOP (6 equiv., $266 \mathrm{mg}, 0.6 \mathrm{mmol})$, HOBt (6 equiv., $81 \mathrm{mg}, 0.6 \mathrm{mmol}$ ) and DiPEA (6.5 equiv., $110 \mu \mathrm{L}, 0.65 \mathrm{mmol})$ in NMP (3 mL) was added and the resulting suspention was shaken for $16 \mathrm{~h}$. The resin was finally washed with NMP ( $5 \mathrm{~mL}, 3 \times, 3 \mathrm{~min}$.) to give title compound $\mathbf{3}$.

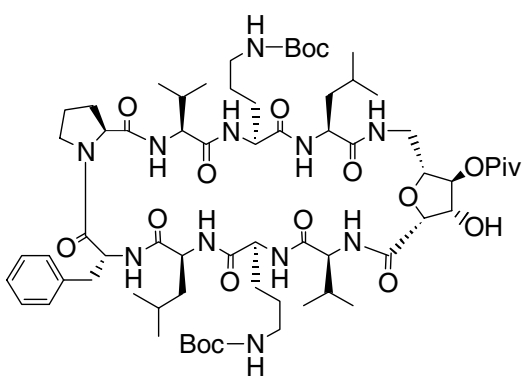

cyclo-[SAA(Piv)-Val-Orn(Boc)-Leu- ${ }^{\mathrm{D}}$ Phe-Pro-Val-Orn(Boc)-Leu] (5) Resin bound nonapeptide 4 was washed with 1,4-dioxane $(5 \mathrm{~mL}, 3 \times, 3$ min.) and taken up in 1,4-dioxane $(10 \mathrm{~mL})$ to which trimethylphosphine (16 equiv., $1.6 \mathrm{~mL}, 1.6 \mathrm{mmol}, 1 \mathrm{M}$ in toluene) was added. Subsequently, the resin was shaken for $2 \mathrm{~h}$., water $(1 \mathrm{~mL})$ was added and shaken another $4 \mathrm{~h}$. The resin was then washed with 1,4-dioxane $(5 \mathrm{~mL}, 3 \times, 3 \mathrm{~min}$.) and DCM $(5 \mathrm{~mL}, 3 \times, 3 \mathrm{~min}$.) after which the peptide was released from the resin by mild acetic cleavage (TFA/DCM, 1/99, v/v, $10 \mathrm{~mL}, 3 \times, 10$ min.). The fractions were collected and coevaporated with toluene (50 $\mathrm{mL}$ ) for three times to give the crude linear peptidic construct which was cyclized directly without further purification.

For the cyclization of the crude linear peptide, it was taken up in DMF ( $5 \mathrm{~mL})$ and added dropwise over the course of an hour to a solution of benzotriazole-1-yl-oxy-tris-pyrrolidino-phosphonium hexafluorophosphate (PyBOP) (5 equiv., $270 \mathrm{mg}, 0.5 \mathrm{mmol}$ ), HOBt (5 equiv., $67 \mathrm{mg}, 0.5 \mathrm{mmol}$ ) and DiPEA (15 equiv., $254 \mu \mathrm{L}$, $1.5 \mathrm{mmol})$ in DMF $(70 \mathrm{~mL})$ and allowed to stir for $16 \mathrm{~h}$. The solvent was removed in vacuo and the resulting 
mixture was applied to a Sephadex® size exclusion colomn $(50.0 \mathrm{mmD} \times 1500 \mathrm{mmL})$ and eluted with $\mathrm{MeOH}$ yielding pure peptide 5 as white amorphous solid (128 mg, $96 \mu \mathrm{mol}, 96 \%)$.

${ }^{1} \mathrm{H}-\mathrm{NMR}$ (400 MHz, DMSO-D6, $300 \mathrm{~K}$ ): $\delta 9.11$ (bs, $1 \mathrm{H}, \mathrm{NH}{ }^{\mathrm{D}} \mathrm{Phe}$ ), 8.65 (d, 1H, $\mathrm{NH}_{\alpha}$ Orn, $\mathrm{J}_{\mathrm{NH}, \mathrm{H}_{\alpha}}=8.8 \mathrm{~Hz}$ ), $8.57\left(\mathrm{~d}, 1 \mathrm{H}, \mathrm{NH}_{\alpha}\right.$ Orn, $\left.\mathrm{J}_{\mathrm{NH}, \mathrm{H}_{\alpha}}=8.6 \mathrm{~Hz}\right), 8.29\left(\mathrm{~d}, 1 \mathrm{H}, \mathrm{NH}\right.$ Leu, $\left.\mathrm{J}_{\mathrm{NH}, \mathrm{H}_{\alpha}}=8.7 \mathrm{~Hz}\right), 7.97\left(\mathrm{~d}, 1 \mathrm{H}, \mathrm{NH} \mathrm{Leu,} \mathrm{J}_{\mathrm{NH}, \mathrm{H}_{\alpha}}=\right.$ $7.6 \mathrm{~Hz}), 7.42$ (m, 2H, NH SAA, NH Val), 7.28 (m, 1H, NH Val), 7.27-7.13 (m, 5H, $\mathrm{H}_{\mathrm{ar}}$ ), 6.86 (bs, 1H, $\mathrm{NH}_{\delta}$ Orn), 6.55 (bs, 1H, $\left.\mathrm{NH}_{\delta} \mathrm{Orn}\right), 5.90\left(\mathrm{~d}, 1 \mathrm{H}, \mathrm{C}_{3}-\mathrm{OH} \mathrm{SAA}, \mathrm{J}_{\mathrm{OH}, \mathrm{H} 3}=4.8 \mathrm{~Hz}\right), 4.87$ (m, 1H, $\mathrm{H}_{\alpha}$ Orn), 4.73 (bs, 1H, $\left.\mathrm{H}_{2} \mathrm{SAA}\right), 4.63$ (m, 1H, $\left.\mathrm{H}_{\alpha} \mathrm{Leu}\right), 4.53$ (m, 1H, $\mathrm{H}_{\alpha}$ Pro), 4.46 (m, 1H, $\mathrm{H}_{\alpha}$ Val), 4.37 (d, 1H, $\left.\mathrm{H}_{4} \mathrm{SAA} \mathrm{J}=3.3 \mathrm{~Hz}\right)$, 4.33 (m, 1H, $\left.\mathrm{H}_{\alpha}{ }^{\mathrm{D}} \mathrm{Phe}\right), 4.22\left(\mathrm{~m}, 2 \mathrm{H}, \mathrm{H}_{\alpha}\right.$ Val, $\mathrm{H}_{\alpha}$ Orn), 4.16 (m, 3H, $\mathrm{H}_{\alpha}$ Leu, $\mathrm{H}_{3}$ SAA, $\left.\mathrm{H}_{5} \mathrm{SAA}\right), 3.51(\mathrm{~m}, 1 \mathrm{H}$, $\mathrm{H}_{\delta^{\mathrm{d}}}$ Pro), 3.39 (m, 1H, $\left.\mathrm{H}_{6 \mathrm{~d}} \mathrm{SAA}\right), 3.28$ (m, 1H, $\left.\mathrm{H}_{6 \mathrm{u}} \mathrm{SAA}\right), 2.98-2.78\left(\mathrm{~m}, 6 \mathrm{H}, \mathrm{H}_{\beta}{ }^{\mathrm{D}} \mathrm{Phe}, \mathrm{H}_{\delta}\right.$ Orn), $2.42(\mathrm{~m}, 1 \mathrm{H}$, $\mathrm{H}_{\delta \mathrm{u}}$ Pro), 2.00 (m, 4H, $\mathrm{H}_{\beta}$ Val, $\mathrm{H}_{\beta}$ Pro), 1.75-1.25 (m, 16H, $\mathrm{H}_{\beta}$ Orn, $\mathrm{H}_{\gamma}$ Orn, $\mathrm{H}_{\beta}$ Leu, $\mathrm{H}_{\gamma}$ Leu, $\mathrm{H}_{\gamma}$ Pro), 1.33 (s, $18 \mathrm{H}, \mathrm{CH}_{3}$ Boc) 1.14 (s, 9H, $\mathrm{CH}_{3}$ Piv) 0.91-0.66 (m, 24H, $\mathrm{H}_{\gamma}$ Val, $\mathrm{H}_{\delta}$ Leu). ATR-IR (thin film) 3274.9, 2960.5, 2931.6, 2873.7, 1633.6, 1525.6, 1450.4, 1390.6, 1365.5, 1276.8, 1251.7, 1164.9, 1093.6, 1037.6, 910.3, 729.0, 702.0, 646.1 $\mathrm{cm}^{-1}$. MS (ESI): $\mathrm{m} / \mathrm{z} 1341.0 \quad[\mathrm{M}+\mathrm{H}]^{+}, \quad 1363.0 \quad[\mathrm{M}+\mathrm{Na}]^{+}$HRMS: calcd for $\mathrm{C}_{67} \mathrm{H}_{109} \mathrm{~N}_{11} \mathrm{O}_{17} \mathrm{NH}_{4}$ 1357.8347, found 1357.8325.

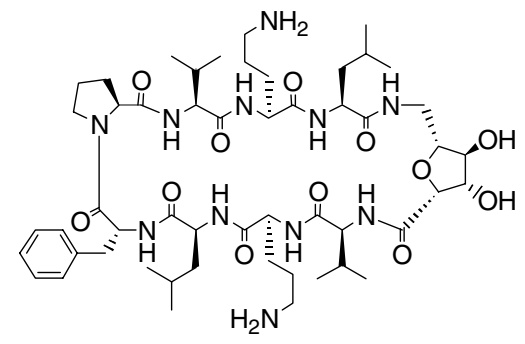

\section{cyclo-[SAA-Val-Orn-Leu- ${ }^{\mathrm{D}}$ Phe-Pro-Val-Orn-Leu] (7)}

Cyclic peptide 5 (64 mg, $48 \mu \mathrm{mol}$ ) was taken up in anhydrous $\mathrm{MeOH}(2.5$ $\mathrm{mL}$ ), sodium methoxide (7.7 equiv., $20 \mathrm{mg}, 370 \mu \mathrm{mol}$ ) was added and the mixture was allowed to stir for $16 \mathrm{~h}$. Subsequently, the solution was neutralized using Amberlite ${ }^{\circledast}$ exchange resin $\left(\mathrm{H}^{+}\right.$-form) and concentrated in vacuo. A portion of the deprotected cyclic peptide $6(36 \mathrm{mg}, 29 \mu \mathrm{mol})$ was directly dissolved in DCM $(5 \mathrm{~mL})$ and cooled to $0{ }^{\circ} \mathrm{C}$. Then, trifluoroacetic acid $(5 \mathrm{~mL})$ was added and the mixture was allowed to warm to ambient temperature over a period of $30 \mathrm{~min}$. To the solution was added toluene $(15 \mathrm{~mL})$ and concentrated. The resulting peptide was analyzed by LC/MS $\left(\mathrm{R}_{\mathrm{t}} 17.56 \mathrm{~min}\right.$, linear gradient $20 \rightarrow 60 \% \mathrm{~B}$ in 20 $\min ; m / z=1057.1[\mathrm{M}+\mathrm{H}]^{+}, 529.1[\mathrm{M}+\mathrm{H}]^{2+}$ ) and purified by semi-preperative RP-HPLC (linear gradient of 4.0 CV; $\left.30 \rightarrow 50 \% \mathrm{~B} ; \mathrm{R}_{\mathrm{t}} 4.0 \mathrm{CV}\right)$. Lyophilization of the combined fractions furnished title compound 7 (22.0 $\mathrm{mg}, 17.1 \mu \mathrm{mol}, 59 \%)$ as white amorphous powder. ${ }^{1} \mathrm{H}-\mathrm{NMR}\left(600 \mathrm{MHz}, \mathrm{CD}_{3} \mathrm{OH}\right): \delta 8.95\left(\mathrm{~d}, 1 \mathrm{H}, \mathrm{NH}^{\mathrm{D}} \mathrm{Phe}_{5}\right.$, $\left.\mathrm{J}_{\mathrm{NH}, \mathrm{H}_{\alpha}}=3.3 \mathrm{~Hz}\right), 8.64\left(\mathrm{~d}, 1 \mathrm{H}, \mathrm{NH} \mathrm{Leu}{ }_{4}, \mathrm{~J}_{\mathrm{NH}, \mathrm{H}_{\alpha}}=9.1 \mathrm{~Hz}\right), 8.62\left(\mathrm{~d}, 2 \mathrm{H}, \mathrm{NH}_{\alpha} \operatorname{Orn}_{3,8}, \mathrm{~J}_{\mathrm{NH}, \mathrm{H}_{\alpha}}=8.7 \mathrm{~Hz}\right), 8.33(\mathrm{~d}, 1 \mathrm{H}$, $\left.\mathrm{NH} \mathrm{Leu}_{9}, \mathrm{~J}_{\mathrm{NH}, \mathrm{H}_{\alpha}}=8.5 \mathrm{~Hz}\right), 8.00\left(\mathrm{t}, 1 \mathrm{H}, \mathrm{NH} \mathrm{SAA}_{1}, \mathrm{~J}_{\mathrm{NH}, 6}=5.3 \mathrm{~Hz}\right), 7.83\left(\mathrm{bs}, 2 \mathrm{H}, \mathrm{NH}_{\delta} \mathrm{Orn}_{3}\right), 7.80\left(\mathrm{bs}, 2 \mathrm{H}, \mathrm{NH}_{\delta}\right.$ $\left.\mathrm{Orn}_{8}\right), 7.77\left(\mathrm{~d}, 1 \mathrm{H}, \mathrm{NH} \mathrm{Val}{ }_{7}, \mathrm{~J}_{\mathrm{NH}, \mathrm{H}_{\alpha}}=8.7 \mathrm{~Hz}\right), 7.46\left(\mathrm{~d}, 1 \mathrm{H}, \mathrm{NH} \mathrm{Val}_{2}, \mathrm{~J}_{\mathrm{NH}, \mathrm{H}_{\alpha}}=8.8 \mathrm{~Hz}\right), 7.40-7.21\left(\mathrm{~m}, 5 \mathrm{H}, \mathrm{H}_{\mathrm{ar}}\right)$, $4.99\left(\mathrm{~m}, 1 \mathrm{H}, \mathrm{H}_{\alpha} \mathrm{Orn}_{3}\right), 4.67\left(\mathrm{~m}, 1 \mathrm{H}, \mathrm{H}_{\alpha} \mathrm{Orn}_{8}\right), 4.63\left(\mathrm{~m}, 1 \mathrm{H}, \mathrm{H}_{\alpha} \mathrm{Leu}_{4}\right), 4.53\left(\mathrm{~d}, 1 \mathrm{H}, \mathrm{H}_{2} \mathrm{SAA}_{1}, \mathrm{~J}_{2,3}=3.9 \mathrm{~Hz}\right)$, $4.48\left(\mathrm{~m}, 1 \mathrm{H}, \mathrm{H}_{\alpha}{ }^{\mathrm{D}} \mathrm{Phe}_{5}\right), 4.46\left(\mathrm{~m}, 1 \mathrm{H}, \mathrm{H}_{\alpha} \mathrm{Leu}_{9}\right), 4.33\left(\mathrm{~m}, 1 \mathrm{H}, \mathrm{H}_{\alpha}\right.$ Pro $\left._{6}\right), 4.30$ (m, 1H, H $\left.\mathrm{Val}_{2}\right), 4.20\left(\mathrm{dd}, 1 \mathrm{H}, \mathrm{H}_{3}\right.$ $\left.\mathrm{SAA}_{1}, \mathrm{~J}_{3,4}=1.6 \mathrm{~Hz}, \mathrm{~J}_{3,2}=3.9 \mathrm{~Hz}\right), 4.10\left(\mathrm{~m}, 1 \mathrm{H}, \mathrm{H}_{5} \mathrm{SAA}_{1}\right), 4.03\left(\mathrm{~m}, 1 \mathrm{H}, \mathrm{H}_{\alpha} \mathrm{Val}_{7}\right), 3.93\left(\mathrm{dd}, 1 \mathrm{H}, \mathrm{H}_{4} \mathrm{SAA}_{1}, \mathrm{~J}_{4,3}\right.$ $\left.=1.6 \mathrm{~Hz}, \mathrm{~J}_{4,5}=1.6 \mathrm{~Hz}\right), 3.72\left(\mathrm{~m}, 1 \mathrm{H}, \mathrm{H}_{\delta \mathrm{d}} \mathrm{PrO}_{6}\right), 3.59\left(\mathrm{ddd}, 1 \mathrm{H}, \mathrm{H}_{6 \mathrm{~d}} \mathrm{SAA}_{1}, \mathrm{~J}_{6 \mathrm{~d}, 5}=3.8 \mathrm{~Hz}, \mathrm{~J}_{6 \mathrm{~d}, \mathrm{NH}}=5.3 \mathrm{~Hz}, \mathrm{~J}_{6 \mathrm{~d}, 6 \mathrm{u}}=\right.$ $14.3 \mathrm{~Hz}), 3.44\left(\mathrm{ddd}, 1 \mathrm{H}, \mathrm{H}_{6 \mathrm{u}} \mathrm{SAA}_{1}, \mathrm{~J}_{6 \mathrm{u}, \mathrm{NH}}=5.3 \mathrm{~Hz}, \mathrm{~J}_{6 \mathrm{u}, 6 \mathrm{~d}}=14.3 \mathrm{~Hz}\right), 3.08\left(\mathrm{dd}, 1 \mathrm{H}, \mathrm{H}_{\beta} \mathrm{d}^{\mathrm{D}} \mathrm{Phe}_{5}, \mathrm{~J}_{\beta \mathrm{d}, \beta \mathrm{u}}=12.6 \mathrm{~Hz}\right.$, $\left.\mathrm{J}_{\beta^{\mathrm{d}, \alpha}}=5.0 \mathrm{~Hz}\right), 2.99\left(\mathrm{~m}, 1 \mathrm{H}, \mathrm{H}_{\delta^{\mathrm{d}}} \mathrm{Orn}_{3}\right), 2.93\left(\mathrm{~m}, 4 \mathrm{H}, \mathrm{H}_{\delta} \mathrm{Orn}_{8}, \mathrm{H}_{\delta^{\mathrm{u}}} \mathrm{Orn}_{3}, \mathrm{H}_{\beta^{\mathrm{u}}}{ }^{\mathrm{D}} \mathrm{Phe}_{5}\right), 2.47\left(\mathrm{~m}, 1 \mathrm{H}, \mathrm{H}_{\delta^{\mathrm{u}}} \operatorname{Pro}_{6}\right), 2.28$ $\left(\mathrm{m}, 1 \mathrm{H}, \mathrm{H}_{\beta} \mathrm{Val}_{7}\right), 2.07\left(\mathrm{~m}, 1 \mathrm{H}, \mathrm{H}_{\beta} \mathrm{Val}_{2}\right), 1.98\left(\mathrm{~m}, 2 \mathrm{H}, \mathrm{H}_{\beta} \mathrm{d} \operatorname{Pro}_{6}, \mathrm{H}_{\beta} \mathrm{d} \operatorname{Orn}_{3}\right), 1.84\left(\mathrm{~m}, 1 \mathrm{H}, \mathrm{H}_{\beta} \mathrm{d} \mathrm{Orn}_{8}\right), 1.76(\mathrm{~m}$,

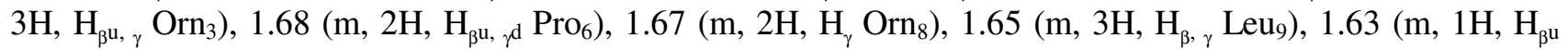
$\left.\mathrm{Orn}_{8}\right), 1.57\left(\mathrm{~m}, 1 \mathrm{H}, \mathrm{H}_{\gamma^{\mathrm{u}}} \mathrm{Pro}_{6}\right), 1.54\left(\mathrm{~m}, 2 \mathrm{H}, \mathrm{H}_{\beta} \mathrm{d}, \gamma=\mathrm{Leu}_{4}\right), 1.40$ (m, 1H, H $\left.\beta_{\beta^{\mathrm{u}}} \mathrm{Leu}_{4}\right), 0.95\left(\mathrm{~m}, 3 \mathrm{H}, \mathrm{H}_{\gamma^{\mathrm{d}}} \mathrm{Val}_{7}\right), 0.93$ $\left(\mathrm{m}, 6 \mathrm{H}, \mathrm{H}_{\gamma} \mathrm{Val}_{2}\right), 0.89\left(\mathrm{~m}, 6 \mathrm{H}, \mathrm{H}_{\delta} \mathrm{Leu}_{4}\right), 0.87\left(\mathrm{~m}, 3 \mathrm{H}, \mathrm{H}_{\gamma^{\mathrm{u}}} \mathrm{Val}_{7}\right), 0.85\left(\mathrm{~m}, 6 \mathrm{H}, \mathrm{H}_{\delta} \mathrm{Leu}_{9}\right)$. The amide region of the ROESY-experiment is depicted in Figure 1. ATR-IR (thin film) 3270.0, 3066.7, 2958.7, 2935.1, 2874.8, 1733.9, 1670.4, 1639.2, 1533.3, 1456.3, 1202.2, 1181.2, 1133.5, 1033.3, 837.4, 799.5, 748.6, 722.4, 702.4 $\mathrm{cm}^{-1}$. HRMS: calcd for $\mathrm{C}_{52} \mathrm{H}_{85} \mathrm{~N}_{11} \mathrm{O}_{12} \mathrm{H} 1056.6457$, found 1056.6382 . 

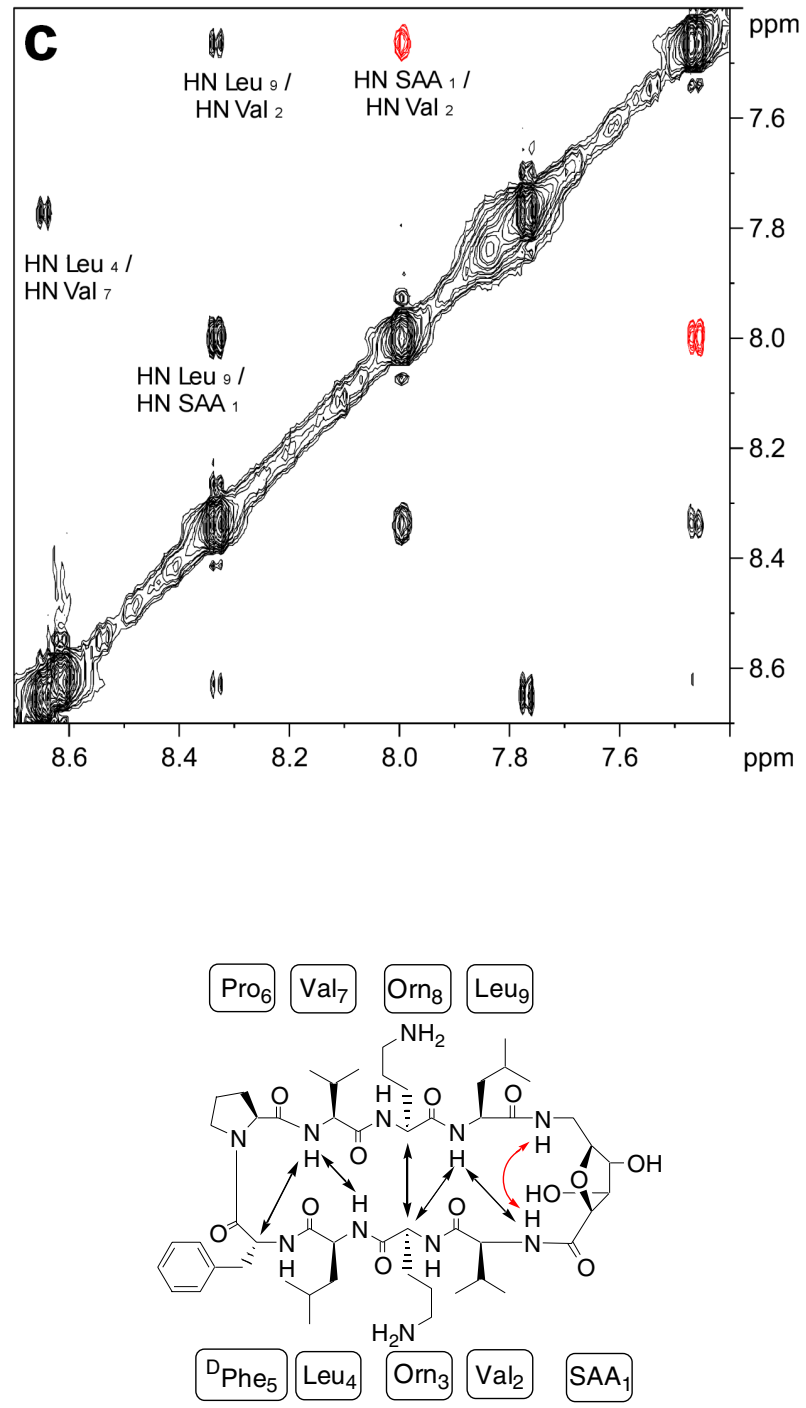

Figure 1 :Amide region of the ROESY experiment of $7\left(\mathrm{CD}_{3} \mathrm{OH}, 600 \mathrm{MHz}\right)$. 


\section{X-ray crystallographic data}

Lyophilized peptide $7(1,40 \mathrm{mg}, 1.09 \mu \mathrm{mol})$ was dissolved in $200 \mu \mathrm{L} \mathrm{MeOH} / \mathrm{H}_{2} \mathrm{O}(1 / 1$, v/v), after which 5 $\mu \mathrm{L}$ of the solution was injected onto a 96-well microtiter plate that was previously filled with n-decane. To the sample, $1 \mu \mathrm{l}$ spermidine tri- $\mathrm{HCl}(0.1 \mathrm{M})$ was added (addition of $1 \mu \mathrm{l}$ 1,5-diaminopentane di-HCl $(30 \%$ w/v) gave similar results), after which the microtiter plate was covered with a mixture of parafine and silicone oil $(10 / 9, v / v)$ and allowed to stand for a period of 2 weeks. The crystals that formed (Figure 2) were then analyzed and the structure refined (Table 1).

A complete dataset was collected from one crystal $(0.8 \times 0.08 \times 0.04 \mathrm{~mm})$ at $100 \mathrm{~K}$ using a Bruker-Nonius FR591 rotating anode generator equipped with kappa-CCD2000 detector and MONTEL multilayer graded xray optics, $\mathrm{CuK} \alpha$ radiation $\left(\lambda=1.54184 \AA\right.$ ). Data were processed using HKL Denzo and Scalepack. ${ }^{3}$ The structure was solved by direct methods (SIR-97) ${ }^{4}$ and refined with full-matrix least-squares analysis on $\mathrm{F}^{2}$ using SHELXL-97..$^{5}$ Due to the limited resolution of $1.2 \AA$, local disorder and the presence of solvent channels in the crystal, hydrogens were not always added and some atoms were refined at multiple positions. Atoms with occupancies lower than unity, disordered side chains and solvent atoms were refined isotropically. Semiempirical absorption correction from equivalents using SORTAV. ${ }^{6}$ CCDC-216610 contains the supplementary crystallographic data for this paper. These data can be obtained free of charge via www.ccdc.cam.ac.uk/conts/retrieving.html (or from the Cambridge Crystallographic Data Centre, 12 Union Road, Cambridge CB21EZ, UK; fax: (+44) 1223-336-033; or deposit@ccdc.cam.ac.uk).

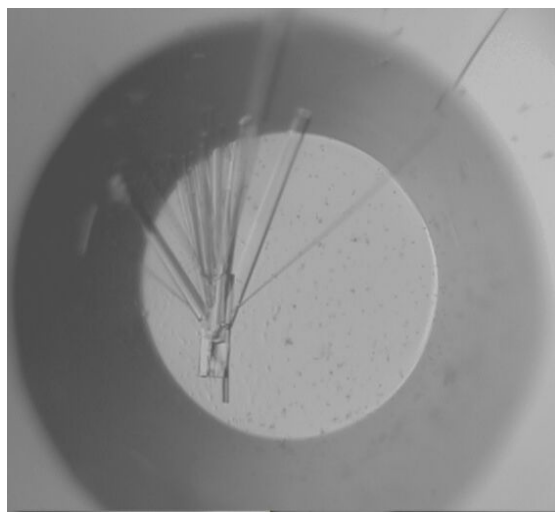

Figure 2: Crystal of GS analogue 7. 
Table 1: Crystal data and structure refinement for GS analogue 7.

\begin{tabular}{|c|c|c|}
\hline Identification code & \multicolumn{2}{|l|}{$\operatorname{gsd} 17 \mathrm{e} 21$} \\
\hline Empirical formula & \multicolumn{2}{|c|}{$\mathrm{C}_{52} \mathrm{H}_{85} \mathrm{~N}_{11} \mathrm{O}_{12}$ 5.75( $\left(\mathrm{H}_{2} \mathrm{O}\right)$} \\
\hline Formula weight & \multicolumn{2}{|c|}{1149.64} \\
\hline Temperature & \multicolumn{2}{|l|}{$100 \mathrm{~K}$} \\
\hline Wavelength & \multicolumn{2}{|l|}{$1.5418 \AA$} \\
\hline Crystal system, & \multicolumn{2}{|l|}{ Hexagonal } \\
\hline Space group & \multicolumn{2}{|c|}{ P6 } \\
\hline \multirow[t]{2}{*}{ Unit cell dimensions } & $\mathrm{a}=31.3930(4) \AA$ & $\alpha=90^{\circ}$ \\
\hline & $\mathrm{b}=31.3930(4) \AA$ & $\beta=90^{\circ}$ \\
\hline Volume & $\begin{array}{l}c=12.7243(2) \AA \\
10860.0(3) \AA^{3}\end{array}$ & $\gamma=120^{\circ}$ \\
\hline $\mathrm{Z}$ & \multicolumn{2}{|l|}{6} \\
\hline Calculated density & \multicolumn{2}{|l|}{$1.055 \mathrm{Mg} / \mathrm{m}^{3}$} \\
\hline Absorption coefficient & \multicolumn{2}{|l|}{$0.666 \mathrm{~mm}^{-1}$} \\
\hline $\mathrm{F}(000)$ & \multicolumn{2}{|l|}{3700} \\
\hline Crystal size & \multicolumn{2}{|c|}{$0.8 \times 0.08 \times 0.04 \mathrm{~mm}$} \\
\hline Theta range for data collection & \multicolumn{2}{|c|}{$3.47^{\circ}$ to $50.32^{\circ}$} \\
\hline Limiting indices & \multicolumn{2}{|l|}{$0<=\mathrm{h}<=27$} \\
\hline & \multicolumn{2}{|l|}{$0<=\mathrm{k}<=26$} \\
\hline & \multicolumn{2}{|l|}{$-12<=\mathrm{l}<=11$} \\
\hline Reflections collected / unique & \multicolumn{2}{|c|}{$49791 / 7378[\mathrm{R}(\mathrm{int})=0.075]$} \\
\hline Completeness to theta $=50.32^{\circ}$ & \multicolumn{2}{|c|}{$99 \%$} \\
\hline Absorption correction & \multicolumn{2}{|c|}{ Semi-empirical from equivalents } \\
\hline Max. and min. transmission & \multicolumn{2}{|c|}{0.980 and 0.911} \\
\hline Refinement method & \multicolumn{2}{|c|}{ Full-matrix least-squares on $\mathrm{F}^{2}$} \\
\hline Data / restraints / parameters & \multicolumn{2}{|c|}{7378 / 38 / 709} \\
\hline Goodness-of-fit on $\mathrm{F}^{2}$ & \multicolumn{2}{|l|}{1.072} \\
\hline Final $R$ indices $\left(\mathrm{F}_{0}>4 \sigma\left(\mathrm{F}_{\mathrm{o}}\right)\right.$ & \multicolumn{2}{|c|}{$\mathrm{R} 1=0.0982, \mathrm{wR} 2=0.2423$} \\
\hline $\mathrm{R}$ indices (all data) & \multicolumn{2}{|c|}{$\mathrm{R} 1=0.1093, \mathrm{wR} 2=0.2590$} \\
\hline Absolute structure parameter & \multicolumn{2}{|c|}{$0.0(4)$} \\
\hline Extinction coefficient & \multicolumn{2}{|l|}{$0.0021(2)$} \\
\hline Largest diff. peak and hole & \multicolumn{2}{|c|}{0.624 and $-0.549 \mathrm{e}^{-3}$} \\
\hline
\end{tabular}

(1) Timmer, M. S. M.; Verdoes, M.; Sliedregt, L. A. J. M.; van der Marel, G. A.; van Boom, J. H.; Overkleeft, H. S. J. Org. Chem. 2003, 68, 9406 - 9411.

(2) Kaiser, E.; Colescott, R. L.; Bossering, C. D.; Cook, P. I. Anal. Biochem. 1970, 34, 595.

(3) Otwinowski, Z. and Minor, W. "Processing of X-ray Diffraction Data Collected in Oscillation Mode", Methods in Enzymology, Volume 276: Macromolecular Crystallography, Part A 1997, 307-326, Carter, Jr.; C. W. \& Sweet, R. M. Eds., Academic Press.

(4) Altomare, A.; Burla, M. C.; Camalli, M.; Cascarano, G. L.; Giacovazzo, C.; Guagliardi, A.; Moliterni, A.G.G.; Polidori, G. \& Spagna, R. J. Appl. Cryst 1999, 32, 115-119.

(5) Sheldrick, G. M. SHELX97. University of Göttingen, Germany 1997.

(6) Blessing, R. H. Acta Cryst. 1995, A51, 33-38. 